\title{
A New Strategy for Improving the Tracking Performance of Magnetic Levitation System in Maglev Train
}

\author{
Mingda Zhai ${ }^{\oplus}$, Zhiqiang Long * and Xiaolong Li \\ College of Intelligence Science and Technology, National University of Defense Technology, \\ Changsha 410073, China \\ * Correspondence: lzq@maglev.cn
}

Received: 18 July 2019; Accepted: 13 August 2019; Published: 16 August 2019

check for updates

\begin{abstract}
The maglev train is a whole new method of transportation without wheels, consisting of 20 groups of symmetry suspension units. The magnetic levitation system plays a major role in suspending the maglev train stably and following the track quickly with the desired gap. However, vertical track irregularity in the maglev train line has a dreadful effect on the tracking performance of the magnetic levitation system. The investigations carried out by our team have revealed that the fluctuation of the suspension gap becomes more and more serious with increases in running speed. In this paper, a mathematical model with consideration of vertical track irregularity is established. In order to overcome and suppress the fluctuation of the suspension gap, we propose a new strategy which includes installing an accelerometer on the electromagnet to address this problem. This strategy has already been successfully implemented and applied to the suspension controller for a magnetic levitation system in the Changsha maglev express. Real operation data indicates the tracking performance of the magnetic levitation system was obviously improved.
\end{abstract}

Keywords: maglev train; symmetry; magnetic levitation system; vertical track irregularity; tracking performance; strategy

\section{Introduction}

In 1814, the first steam locomotive was invented by George Stephenson, renowned as the "Father of Railways". The Stockton and Darlington Railway was opened to carry passengers in 1825. Rail transport was one of the most important technological inventions of the industrial revolution. Ever since then, the wheel of time has never stopped. Time goes by as fast as a high-speed train, and we have already entered into an age without wheels. The maglev train is a whole new method of transportation without wheels. Plenty of scholars and engineers in Japan, the United States, China and South Korea are carrying out research in related fields and have already achieved many successes [1-3]. It is well known that the Shanghai high-speed maglev train went into operation in 2002. The Incheon airport maglev line of South Korea officially began to carry passengers on 3 February 2016. A superconducting maglev train with a high speed of $603 \mathrm{~km} / \mathrm{h}$ will come out in 2027.

As shown in Figure 1, the Changsha maglev express is the first domestic medium-low speed maglev line in China and completely owns the proprietary intellectual property rights. This line stretches over $18.55 \mathrm{~km}$ and started trial operations on 6 May 2016. Beijing's 1st maglev Line S1 is the second domestic medium-low speed maglev line in China and began operating on 31 December 2017. The Qingyuan maglev travel line is under construction, and will go into operation on September 2019. In recent years, the maglev train increasingly began to receive considerable attention and has become an active traffic field. 


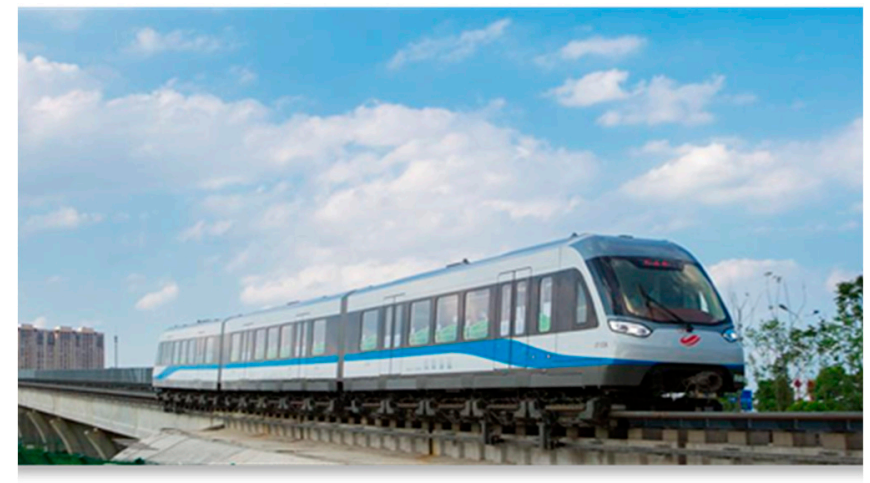

Figure 1. A photograph of the medium-low speed maglev train in Changsha.

Compared with conventional railway systems, the medium-low speed maglev train makes full use of electromagnetic force to achieve active suspension and runs the train using a linear motor. It completely abandons the wheel-rail relationship and achieves non-contact motion, which not only eliminates friction to reduce noise and vibration, but also allows the maglev train to travel through buildings on viaducts smoothly and quietly. What's more, there are zero emissions to the atmosphere during running. Therefore, the maglev train is safe, sound, low-carbon and environmentally friendly.

This paper is concerned chiefly with the study of the magnetic levitation system in a medium-low speed maglev train. The magnetic levitation system plays a major role in suspending the maglev train stably and following the track quickly. Most scholars concentrate on the research on the levitation control algorithm under the conditions of a straight track. However, vertical track irregularity in the maglev train line has a baneful influence on the tracking performance. Unfortunately, many experts are not concerned about this issue. In order to address this issue, the mathematical model with consideration of vertical track irregularity is established in this paper. For the benefit of improving the tracking performance, we propose a new strategy of installing an accelerometer on the electromagnet to address this problem.

\section{Nominal Controller of Magnetic Levitation System}

Magnets of the same polarity repel each other, while those of different polarity attract each other. The medium-low speed maglev train takes advantage of the attraction between the electromagnet and the F-type track to realize suspension at a defined height. A complete maglev train has 20 sets of electromagnets, installed on the bottom of the carriage. When the current is switched on, upward electromagnetic force is generated. The F-type track will suck up the base of the vehicle, and the base and the carriage are connected by air springs. Thus, the maglev train is able to suspend itself in the air, against gravity. The interaction between electromagnetic force and gravity will always keep a dynamic balance, levitating the maglev train above the rail. No matter how the speed and railway line changes, the gap between the track and the electromagnet has to remain unchanged under the action of the suspension controller for the magnetic levitation system.

As shown in Figure 2, the magnetic levitation control system consists of a suspension controller, a gap sensor and an actuator, which is the electromagnet. The gap sensor measures the gap between the electromagnet and the F-type track constantly. The suspension controller calculates the control quantity according to the measured gap signal to adjust the current of the electromagnet in real time. When the current varies, the intensity of the closed-loop magnetic field established between the electromagnet and the F-type track will change accordingly. The electromagnetic force will consequently vary and make the electromagnet fall or float to levitate the maglev train in the target gap. 


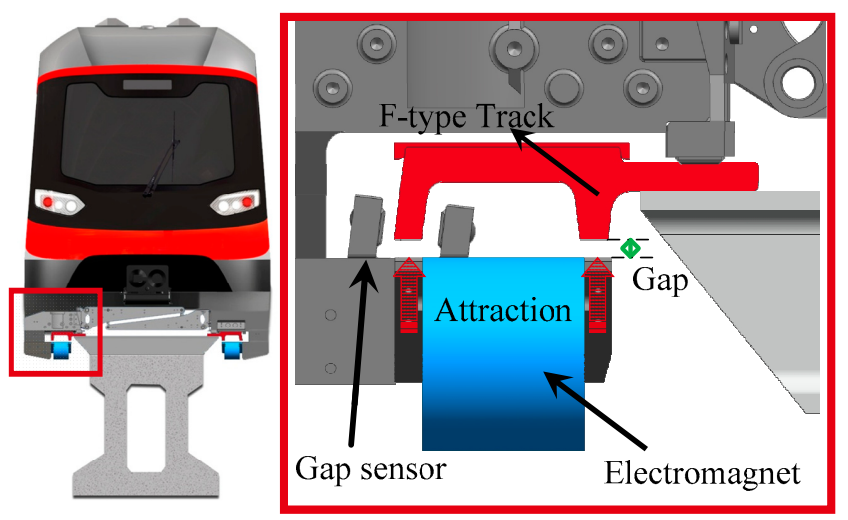

Figure 2. The structure of the magnetic levitation system in the medium-low speed maglev train.

Because of the symmetry of the magnetic levitation system in medium-low speed maglev trains, the mathematical model of single magnetic levitation system is easily established. If all factors are taken into account, the order of the whole model will be very high, which is not conducive to resolving the characteristics of the magnetic levitation system and designing the suspension control algorithm [4]. Therefore, some minor factors are neglected in practice:

- The magnetic flux leakage and edge effect are neglected, and it is determined that the magnetic flux is uniformly distributed in the air gap.

- It is considered that the electromagnetic force provided by the electromagnet concentrates on the geometric center, and the geometric center of the electromagnet coincides with the center of mass.

- There is no dislocation between the magnetic pole surface and the F-type track, namely, there is only vertical motion of the electromagnet relative to the track.

\subsection{Reference Coordinate Equation}

The reference coordinate system XOY is established in Figure 3. $z(t)$ is the absolute displacement of the electromagnet relative to the reference plane and $c(t)$ is the suspension gap between the track and the electromagnet. When the track does not have vertical motion, the following formula is satisfied:

$$
c(t)=z(t)
$$

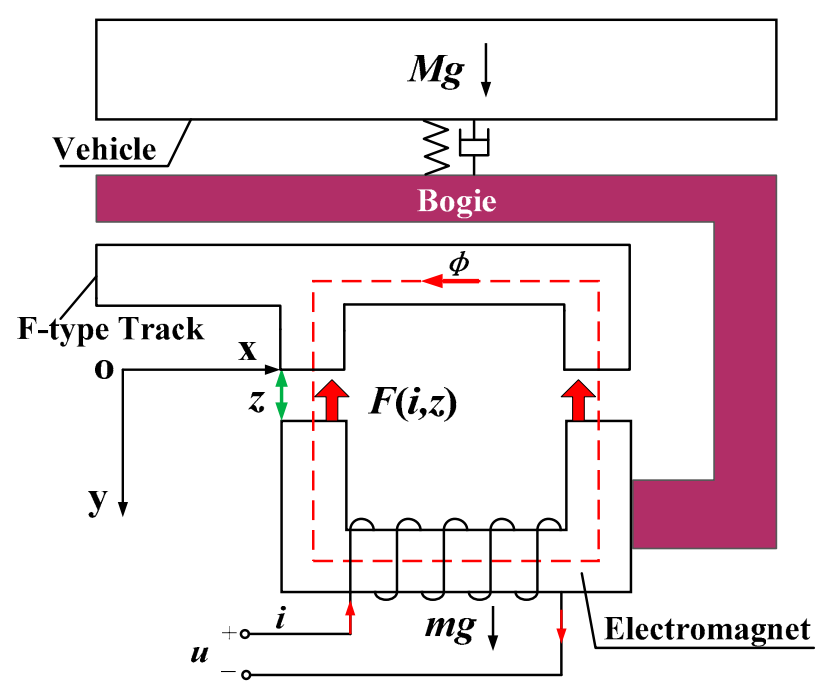

Figure 3. The model of magnetic levitation system in a medium-low speed maglev train. 


\subsection{Electrical Equation}

Since the electromagnet is an inductive component, the electrical equation is:

$$
u(t)=R i(t)+\frac{\mu_{0} N^{2} A}{2} \cdot \frac{\dot{i}(t)}{z(t)}-\frac{\mu_{0} N^{2} A i(t)}{2} \frac{\dot{z}(t)}{[z(t)]^{2}}
$$

In this equation, $u(t)$ is the control voltage applied to both ends of the electromagnet coil, and $R$ is the resistance. The number of turns, the polar area and the permeability of vacuum are $N, A$ and $\mu_{0}$ respectively.

\subsection{Electromagnetic Force Equation}

$$
F(i, z)=\frac{\mu_{0} N^{2} A}{4}\left[\frac{i(t)}{z(t)}\right]^{2}
$$

In this equation, $F(i, z)$ is the electromagnetic force, and $i(t)$ is the current of the electromagnet coil.

\subsection{Kinetic Equation}

$$
m \ddot{z}(t)=(m+M) g-F(i, z)
$$

In this equation, $m$ is the equivalent mass of electromagnet, and $M$ is the equivalent mass of carriage.

Thus, the state variables are specified, and the mathematical model of magnetic levitation system is established:

$$
\begin{aligned}
\mathbf{x}= & \left(\begin{array}{lll}
x_{1} & x_{2} & x_{3}
\end{array}\right)^{T}=\left(\begin{array}{lll}
z & \dot{z} & i
\end{array}\right)^{T} \\
& \left\{\begin{array}{l}
\dot{x}_{1}=x_{2} \\
\dot{x}_{2}=\frac{M+m}{m} g-\frac{K}{m} \frac{x_{3}^{2}}{x_{1}^{2}} \\
\dot{x}_{3}=\frac{x_{2} x_{3}}{x_{1}}-\frac{R x_{1} x_{3}}{2 K}+\frac{x_{1}}{2 K} u
\end{array}\right.
\end{aligned}
$$

where, $K=\mu_{0} A N^{2} / 4$

The parameters of the magnetic levitation system are indicated in Table 1.

Table 1. Parameter list of magnetic levitation system.

\begin{tabular}{cccc}
\hline Symbol & Meaning & Quantity & Unit \\
\hline$m$ & Mass of electromagnet & 400 & $\mathrm{~kg}$ \\
$M$ & Mass of carriage & 1000 & $\mathrm{~kg}$ \\
$R$ & Resistance & 1 & $\Omega$ \\
$N$ & Number of turns & 360 & \\
$A$ & Polar area & 0.038 & $\mathrm{~m}^{2}$ \\
$\mu_{0}$ & vacuum permeability & $4 \pi \times 10^{-7}$ & \\
$z_{0}$ & set gap & 10 & $\mathrm{~mm}$ \\
\hline
\end{tabular}

From the above discussion, it is found that the magnetic levitation system is typically nonlinear. It is very formidable work to analyze the performance of the system by resolving the non-linear differential equation. The linear analysis is often adopted as a solution to address this problem.

Hartman-Grobman theory: If $x_{0}$ is hyperbolic singular point of a nonlinear system, and the following formula is satisfied:

$$
\lim _{x \rightarrow x_{0}}\left(\frac{X\left(x-x_{0}\right)}{\left|x-x_{0}\right|}\right)=0
$$


Therefore, the nonlinear system is determined to have the same topological structure as the corresponding linear system at the isolated singularity $x_{0}$. What's more, if $x_{0}$ is the equilibrium point of the nonlinear system, the stability near the equilibrium point also depends on the corresponding linear system.

For the magnetic levitation system above, the equation at equilibrium position is presented:

$$
(m+M) g=F\left(i_{0}, z_{0}\right)=K \cdot\left[\frac{i_{0}}{z_{0}}\right]^{2}
$$

In this equation, $i_{0}$ is the current of electromagnet coil at equilibrium position, while $z_{0}$ is the suspension gap at equilibrium position. The linearization is accomplished near the equilibrium point, and the linearization model is obtained [5].

$$
\left\{\begin{array}{l}
m \ddot{z}(t)=\left(\frac{2 K i_{0}^{2}}{z_{0}^{3}} \Delta z(t)-\frac{2 K i_{0}}{z_{0}^{2}} \Delta i(t)\right) \\
\Delta u(t)=R \Delta i(t)+\frac{2 K}{z_{0}} \Delta \dot{i}(t)-\frac{2 K i_{0}}{z_{0}^{2}} \Delta \dot{z}(t)
\end{array}\right.
$$

Thus, the state variables of linearization system are specified, and the state space equation of linearization system is defined:

$$
\begin{aligned}
& \mathbf{x}=\left(\begin{array}{lll}
x_{1} & x_{2} & x_{3}
\end{array}\right)^{T}=\left(\begin{array}{lll}
\Delta z & \Delta \dot{z} & \Delta i
\end{array}\right)^{T} \\
& \dot{\mathbf{x}}=\left(\begin{array}{lll}
0 & 1 & 0 \\
\frac{2 K i_{0}{ }^{2}}{z_{0}^{3} m} & 0 & -\frac{2 K i_{0}}{z_{0}{ }^{2} m} \\
0 & \frac{i_{0}}{z_{0}} & -\frac{R z_{0}}{2 K}
\end{array}\right) \mathbf{x}+\left(\begin{array}{l}
0 \\
0 \\
\frac{z_{0}}{2 K}
\end{array}\right) \mathbf{u} \\
& \mathbf{y}=\left(\begin{array}{llll}
1 & 0 & 0 & 0
\end{array}\right) \mathbf{x}
\end{aligned}
$$

The characteristic polynomial is as follows:

$$
\begin{aligned}
|\lambda I-A|=\left|\begin{array}{ccc}
\lambda & -1 & 0 \\
-\frac{2 K i_{0}{ }^{2}}{z_{0}{ }^{3} m} & \lambda & \frac{2 K i_{0}}{z_{0}^{2} m} \\
0 & -\frac{i_{0}}{z_{0}} & \lambda+\frac{R z_{0}}{2 K}
\end{array}\right| \\
=\lambda^{3}+\frac{R z_{0}}{2 K} \lambda^{2}-\frac{R i_{0}^{2}}{z_{0}^{2} m}
\end{aligned}
$$

According to Equation (10), the coefficients of characteristic polynomial fail to satisfy the Hurwitz stability criterion. This linearization system is unstable. Therefore, the magnetic levitation system is unstable according to the Hartman-Grobman theory.

The controllability of the magnetic levitation system is determined by achieving and analyzing the rank of the controllability matrix.

$$
\operatorname{rank}\left(\left[B A B A^{2} B\right]\right)=3
$$

Therefore, the magnetic levitation system is completely controllable. By designing a perfect controller, the magnetic levitation system is able to work steadily in the desired state.

In the field of industrial and traffic control, the most successful and mature control algorithm is the PID algorithm. In our system, the fundamental features of the PID algorithm are reproduced. Firstly, the PID algorithm is less dependent on the accuracy of the model, and is ideal for a nonlinear system. Moreover, the PID algorithm facilitates engineering realization. Consequently, the PID algorithm is the conventional control algorithm in magnetic levitation systems of maglev trains. Previous work has also proved that the PID controller is nominal. A PID controller is not only easy to design, but also conducive to realization in engineering. 
Based on the idea of cascade control, our control algorithm consists of two parts. The inner loop is the current loop and the outer loop is the position loop. When the magnetic levitation system works in the equilibrium position, the inductance of the electromagnet varies in an extremely small range. We refer to it as a constant consequently. Near the equilibrium point, the voltage-current equation of the electromagnet is described as:

$$
u=R \cdot i+\frac{2 K}{z_{0}} \cdot \dot{i}
$$

The voltage and current transfer function of the electromagnet is as follows:

$$
G_{i}(s)=\frac{I(s)}{U(s)}=\frac{1 / R}{\frac{2 K}{R z_{0}} \cdot s+1}
$$

The above formula serves to illustrate that the electromagnet is a typical inertia link, and accordingly the current response seriously lags behind the voltage change. The response time was not able to meet the demand of the maglev train. Therefore, the current feedback method was adopted to reduce the response time in Figure 4.

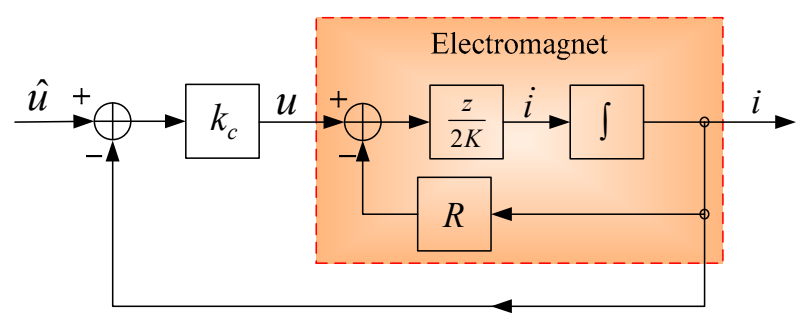

Figure 4. The schematic diagram of current loop.

The input voltage before introducing the current loop was $\hat{u}$, and the voltage acting on the electromagnet is described as:

$$
u=k_{c} \cdot(\hat{u}-i)
$$

The function of negative feedback is to make the output equal to the input. The purpose of preamplifier $k_{c}$ is to speed up response. As shown in Figure 5, the response time of the electromagnet was reduced down to 10 milliseconds. The relationship between the voltage and the current of the electromagnet was settled to be a proportional link in the frequency band of the magnetic levitation system. In addition, the current loop also decreased the order of the magnetic levitation system from three to two.

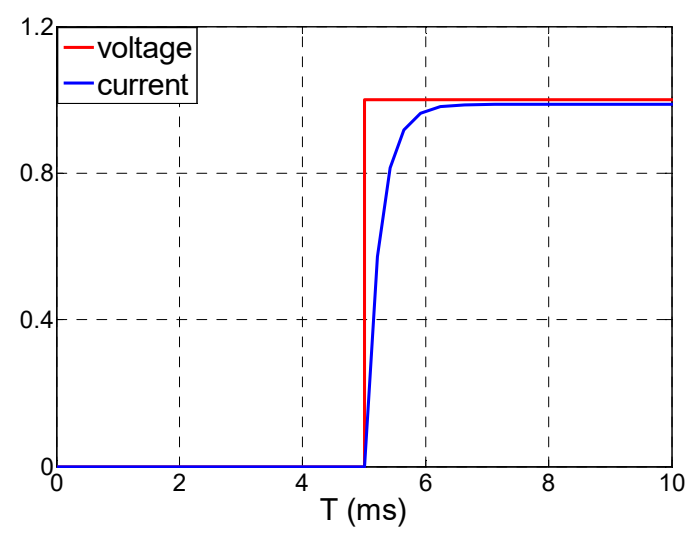

Figure 5. The response of the electromagnet using a current loop. 
The next step was to perform the corresponding position loop.

$$
\hat{u}=k_{p 1}\left(z-z_{0}\right)+k_{d 1} \dot{z}+k_{i 1} \int_{0}^{t}\left(z-z_{0}\right) \mathrm{dt}
$$

Among, $k_{p 1}$ is proportional gain, $k_{d 1}$ is differential gain and $k_{i 1}$ is integral gain. The outer position loop was designed completely.

Equation (15) was brought into Equation (14), and the nominal controller for the magnetic levitation system was designed completely. In order to correspond with the actual engineering signal, the final control law is presented here:

$$
\begin{aligned}
u & =k_{c 1} \cdot\left(k_{p 1}\left(z-z_{0}\right)+k_{d 1} \dot{z}+k_{i 1} \int_{0}^{t}\left(z-z_{0}\right) \mathrm{dt}-k_{c 2} i\right) \\
& =k_{p}\left(z-z_{0}\right)+k_{d} \dot{z}+k_{i} \int_{0}^{t}\left(z-z_{0}\right) \mathrm{dt}-k_{c} i
\end{aligned}
$$

In order to examine the performance of the designed controller, the designed control algorithm was directly substituted into the nonlinear model of the magnetic levitation system in Figure 6. The dynamic characteristics were verified.

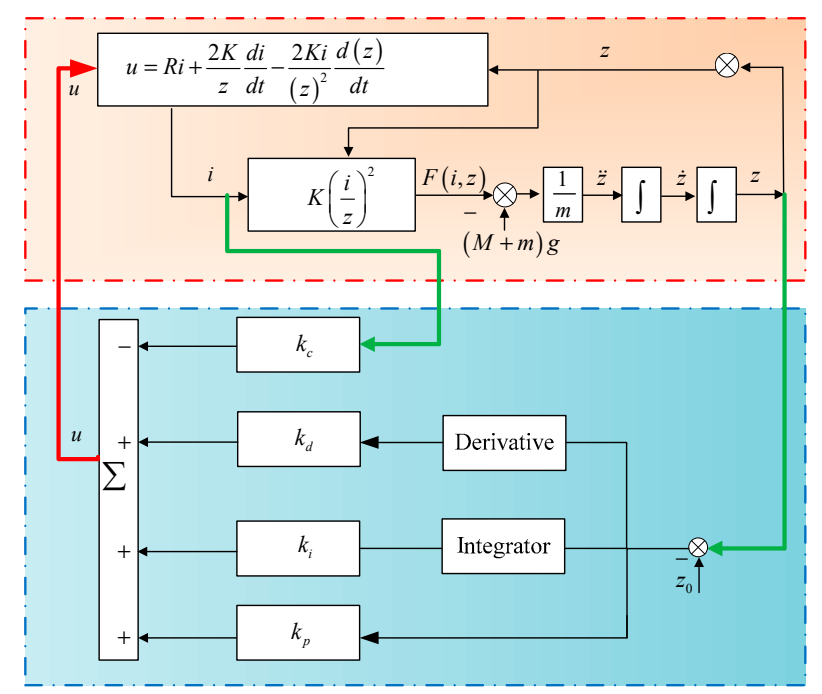

Figure 6. The nominal control block diagram of magnetic levitation system.

The initial gap for the electromagnet was set to $25 \mathrm{~mm}$ and the desired gap was set to $10 \mathrm{~mm}$. The suspension gap of the electromagnet when the floating instruction was issued is presented in Figure 7.

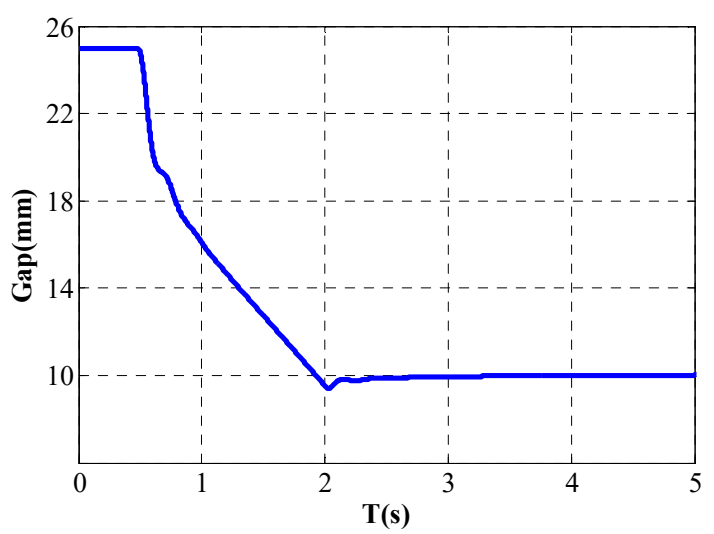

Figure 7. The suspension gap of the electromagnet. 
As shown in Figure 7, the electromagnet began to rise slowly after receiving the command signal. The suspension gap became smaller little by little, and eventually was fixed at the set gap of $10 \mathrm{~mm}$. There was no obvious fluctuation in the whole process, and the system overshoot and response time were within a reasonable range. The simulation result shows the designed controller is able to levitate the maglev train with the desired gap.

\section{The Tracking Performance of Magnetic Levitation System on the Vertical Track Irregularity Condition}

However, due to topographic fluctuations or other spatial constraints, the profile of a maglev train line will change [6-8]. The profile of a line is composed of several gradient lines. If the angles of two adjacent slopes exceed the allowable range, a vertical curve is needed to connect. The vertical curve of a maglev line is usually a circular curve, and there is no transition curve between the straight line and the vertical curve [9-12]. The appearance of a vertical curve is not ideal and is consequently disadvantageous to the magnetic levitation system [13-15]. The tracking performance of a magnetic levitation system with consideration of the vertical curve in a maglev train line needs to be further discussed and analyzed, which plays an important role in the safety and comfort of maglev trains.

As described in Figure 8, the profile of a maglev train line is composed of five parts, the strait section $A B$, the concave curve of section $B C$, the oblique curve of section $C D$, the convex curve of section $D E$ and the strait section $E F$. The radius of circular curve is $r$ and the angle of slope is $\alpha$. The displacement of the track relative to the absolute reference plane is $z_{G}$. It is assumed that the tangent velocity along the track is $V$ and remains constant.

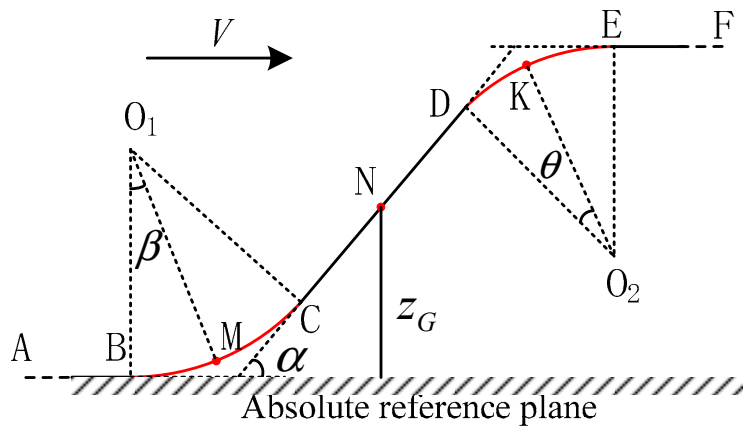

Figure 8. The illustration of the vertical track irregularity in maglev train line.

The strait section $A B$ :

$$
\mathrm{z}_{G}(\mathrm{t})=0
$$

The concave curve of section $B C$ :

$$
\mathrm{z}_{G}(\mathrm{t})=r \cdot(1-\cos \beta)=r \cdot 2\left(\sin \frac{\beta}{2}\right)^{2} \approx r \cdot \frac{\beta^{2}}{2}=\frac{V^{2} \cdot t_{B M}^{2}}{2 r}
$$

In this equation, $\mathrm{M}$ is any point on the concave curve of section $B C$. $\beta$ is very small, so the approximate operation is performed in above formula. $t_{B M}$ is the time spent through the BM segment.

The oblique curve of section $C D$ :

$$
\mathrm{z}_{G}(\mathrm{t})=r \cdot(1-\cos \alpha)+V \cdot t_{C N} \cdot \sin \alpha
$$

In this equation, $\mathrm{N}$ is any point on the oblique curve of section $C D . t_{C N}$ is the time spent through the $\mathrm{CN}$ segment.

The convex curve of section $D E$ :

$$
\mathrm{z}_{G}(\mathrm{t})=r \cdot(1-\cos \alpha)+C D \cdot \sin \alpha+r \cdot \sin \left(\frac{\pi}{2}-\alpha+\frac{V t_{D K}}{r}\right)-r \cdot \cos \alpha
$$


In this equation, $\mathrm{K}$ is any point on the convex curve of section $D E . t_{D K}$ is the time spent through the DK segment.

The strait section EF:

$$
\mathrm{z}_{G}(\mathrm{t})=r \cdot(1-\cos \alpha)+C D \cdot \sin \alpha+r \cdot(1-\cos \alpha)
$$

When the track displacement changes, the suspension gap is no longer equal to the displacement of the electromagnet. In this case, the suspension gap is equal to the difference value between the displacement of the electromagnet and the displacement of the track. Namely:

$$
c(t)=z(t)-z_{G}(\mathbf{t})
$$

Therefore, the model of the magnetic levitation system will change depending on the vertical track irregularity conditions. The electrical equation is defined again:

$$
u=R i+\frac{2 K}{z-z_{G}} \frac{d i}{d t}-\frac{2 K i}{\left(z-z_{G}\right)^{2}} \frac{d\left(z-z_{G}\right)}{d t}
$$

The electromagnetic force equation is become:

$$
F(i, c)=K\left(\frac{i}{z-z_{G}}\right)^{2}
$$

The kinetic equation is presented under these circumstances:

$$
m \ddot{z}(t)=(m+M) g-F(i, c)
$$

However, the controller is unchanged. The function of the controller is to levitate the magnetic levitation system at the defined height. In other words, the suspension gap is controlled so that it will remain invariable under various line conditions. As shown in Figure 9, the above nominal controller was brought into the new nonlinear model with consideration of track irregularity in order to achieve an overall evaluation of tracking performance on the vertical track irregularity condition.

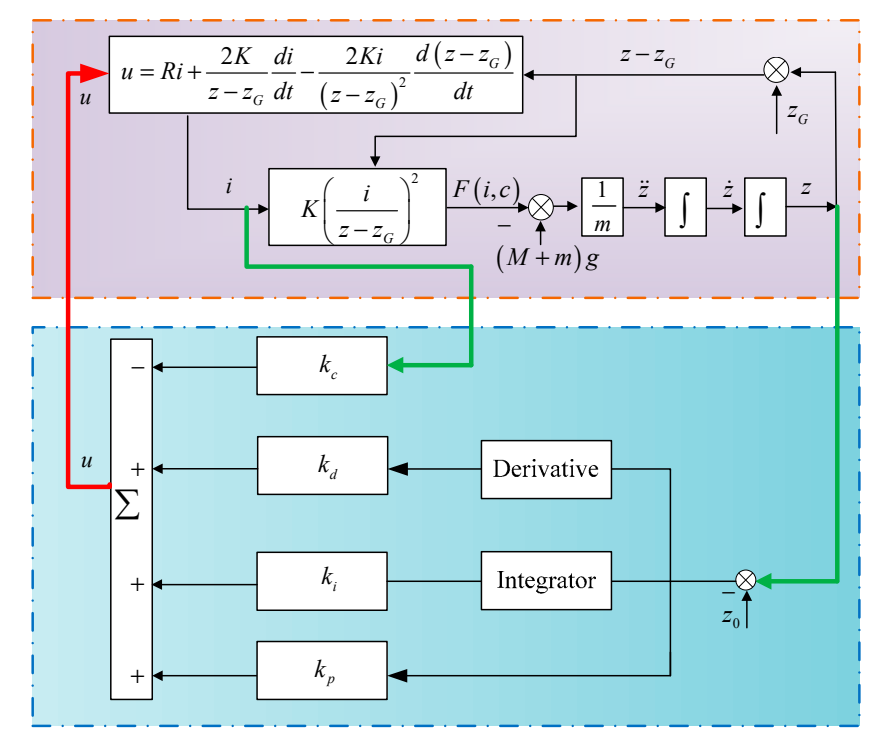

Figure 9. The control block diagram of magnetic levitation system with consideration of track irregularity in maglev train line. 
According to the above analysis, the new model of the magnetic levitation system is closely related to the radius of circular curve $r$ and the angle of slope $\alpha$. Their settings are clearly stipulated in the regulations for track construction of maglev lines, with a minimum radius of $1500 \mathrm{~m}$ and a maximum slope angle of $7 \%$. What's more, the model of the magnetic levitation system also includes the running speed $V$. However, the radius of circular curve and the slope angle are fixed once the maglev train line is built; the investigation of the relationship of tracking performance and speed makes more sense.

When the maglev train passes through the track shown in Figure 8 at a certain speed, the magnetic levitation system will go through four different stages: entering the concave curve stage, withdrawing from the concave curve stage, entering the convex curve stage and withdrawing from the convex curve stage. In order to discuss the tracking performance of the magnetic levitation system at different speeds, three representative speeds were selected to observe the tracking performance on the above four stages.

As described in Figure 10, the tracking performance of a magnetic levitation system when passing through the vertical track irregularity in a maglev train line was investigated and achieved. The suspension gap will fluctuate upward or downward, and stabilize in the set gap after a period of time. It is apparent that the fluctuation of the suspension gap becomes more and more serious with increases in speed. At some stages, the tracking performance of the magnetic levitation system is extremely awful and unacceptable, and the electromagnet is prone to collide with the F-type track.
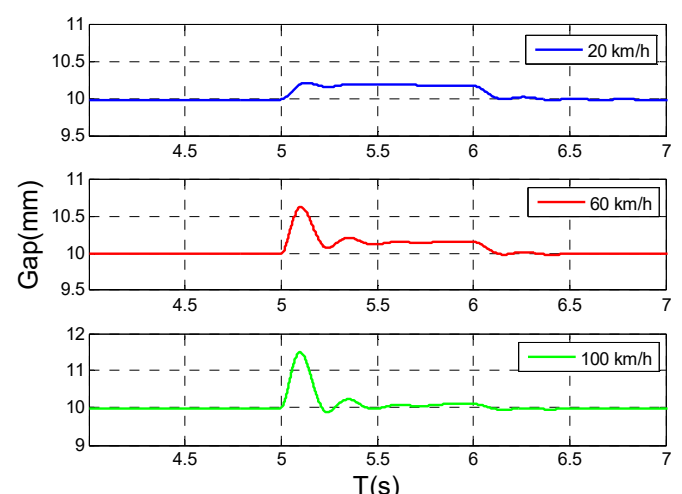

(a)
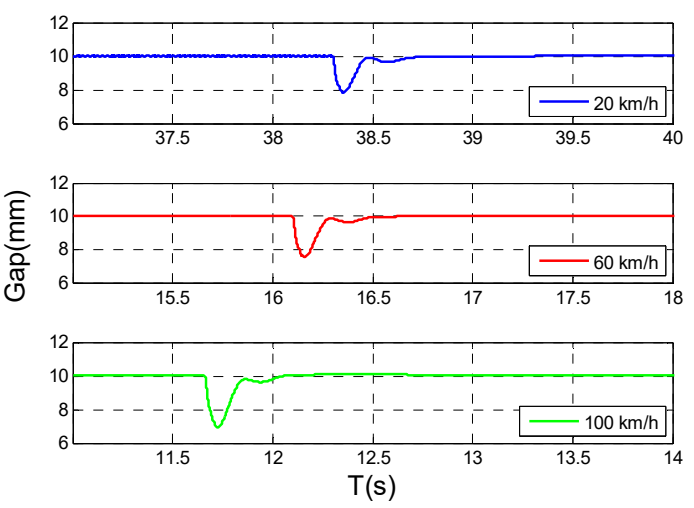

(c)
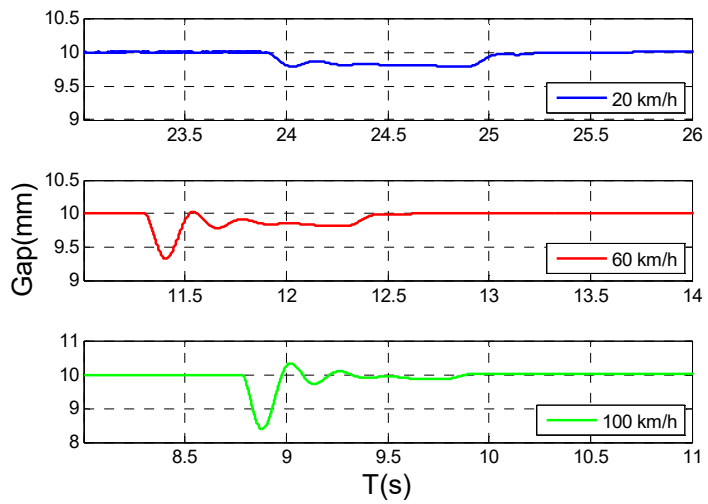

(b)
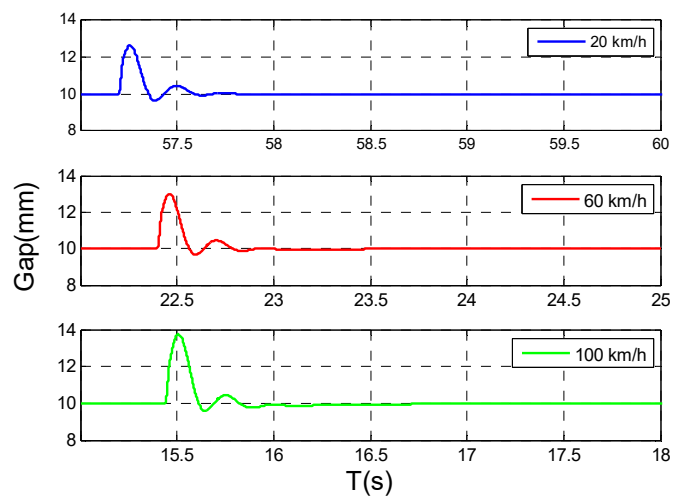

(d)

Figure 10. The tracking performance of magnetic levitation system when passing through the vertical track irregularity in a maglev train line. (a) The suspension gap when entering the concave curve stage; (b) the suspension gap when withdrawing from the concave curve stage; (c) the suspension gap when entering the convex curve stage; (d) the suspension gap when withdrawing from the convex curve stage. 
In order to verify the rationality of the above analysis, we conduct a series of simulations and investigations to achieve the relationship of the maximum fluctuations of suspension gap and speeds. The maximum fluctuation of the suspension gap at five different speeds is shown in Figure 11.

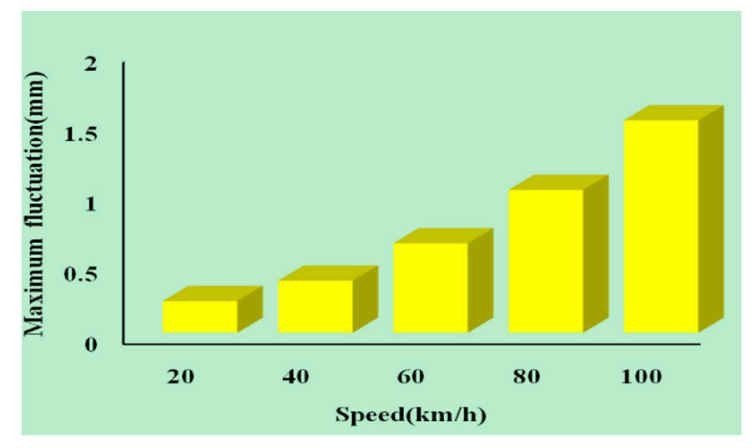

(a)

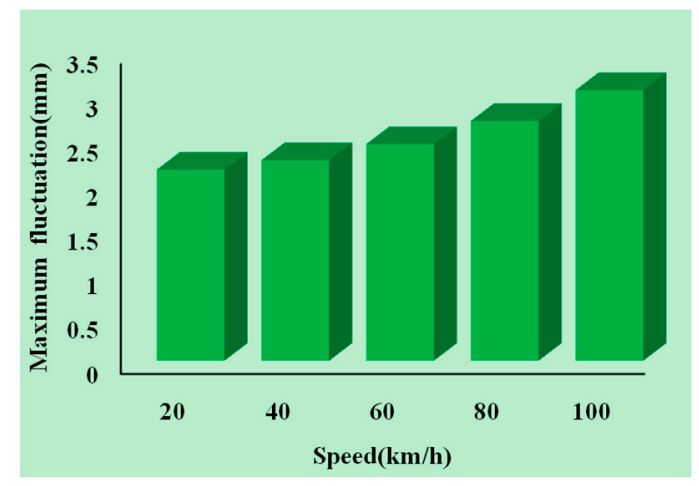

(c)

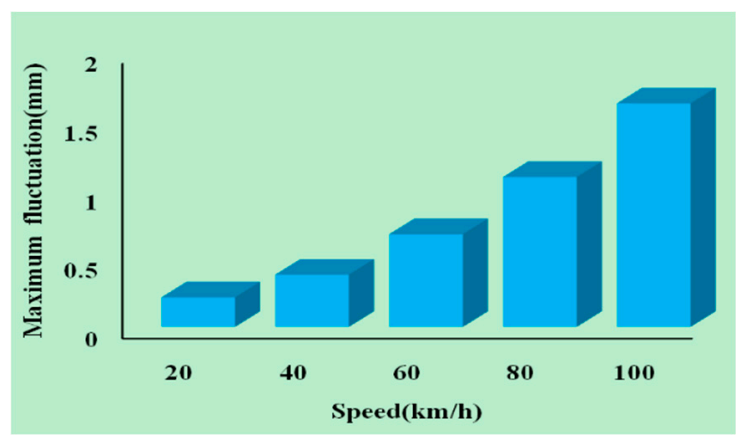

(b)

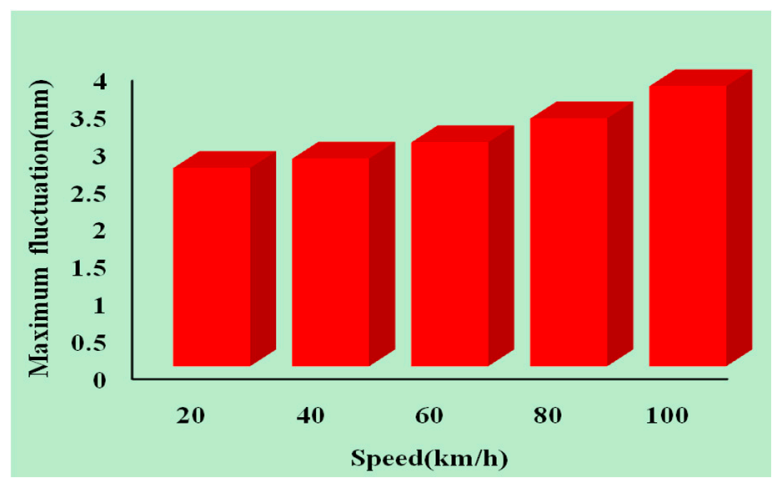

(d)

Figure 11. The maximum fluctuation of the suspension gap when passing through the vertical track irregularity in maglev train line. (a) Entering the concave curve stage; (b) withdrawing from the concave curve stage; (c) entering the convex curve stage; (d) withdrawing from the convex curve stage.

Taking into account the above analysis and discussion, a number of significant conclusions were acquired. It is clear that the fluctuation of the suspension gap becomes more and more serious with the increase of speed in the above four stages. Under high-speed conditions, the fluctuation of the suspension gap is too violent. When withdrawing from the convex curve stage, the maximum fluctuation at a speed of $100 \mathrm{~km} / \mathrm{h}$ is up to $3.7532 \mathrm{~mm}$, and the electromagnet is prone to collide with the F-type track. In other words, the tracking performance of the nominal controller is unpleasant.

\section{A New Strategy for Improving the Tracking Performance of the Magnetic Levitation System}

Although the designed nominal controller is able to make the maglev train stay within in the target gap, the tracking performance is not ideal for the vertical track irregularity condition. What's more, the fluctuation of the suspension gap is too violent, and the electromagnet is prone to collide with the F-type track at some stages. Therefore, the existing control strategy is not satisfied on the vertical track irregularity condition. We propose a new strategy by installing an accelerometer on the electromagnet as a solution to address this problem.

In the magnetic levitation system, the existing gap sensor can only measure and supply gap signals. The suspension controller is not enough, especially when the magnetic levitation system passes through the vertical track irregularly in a maglev train line. At the same time, the vertical acceleration of the electromagnet will change above four stages. Therefore, we propose to improve the tracking performance of the magnetic levitation system by obtaining and utilizing the acceleration of the electromagnet. 
However, when the maglev train passes through the vertical curve, the data measured by the accelerometer includes not only the gravity acceleration component, but also the centrifugal acceleration component. Therefore, for the vertical curve, it is necessary to analyze the measurements of the accelerometer $a(t)$ separately.

The strait section $A B$ :

$$
a(t)=g-\ddot{z}(t)
$$

The concave curve of section $B C$ :

$$
a(t)=g \cos \theta+\frac{v^{2}}{r}-\ddot{z}(t)
$$

The oblique curve of section $C D$ :

$$
a(t)=g \cos \theta_{0}-\ddot{z}(t)
$$

The convex curve of section $D E$ :

$$
a(t)=g \cos \theta-\frac{v^{2}}{r}-\ddot{z}(t)
$$

The strait section $E F$ :

$$
a(t)=g-\ddot{z}(t)
$$

Therefore, the measured acceleration signals were not able to be directly used in the design of the controller. The acceleration signal must be pre-processed. The low-frequency signals, such as the gravity acceleration component and centrifugal acceleration, are filtered, and the dynamic and vertical high-frequency signals of electromagnet are retained. The high-pass filter is used to filter and extract signals.

In summary, the new control block diagram of the magnetic levitation system was achieved. Consideration of vertical curves in a maglev train line is presented in Figure 12.

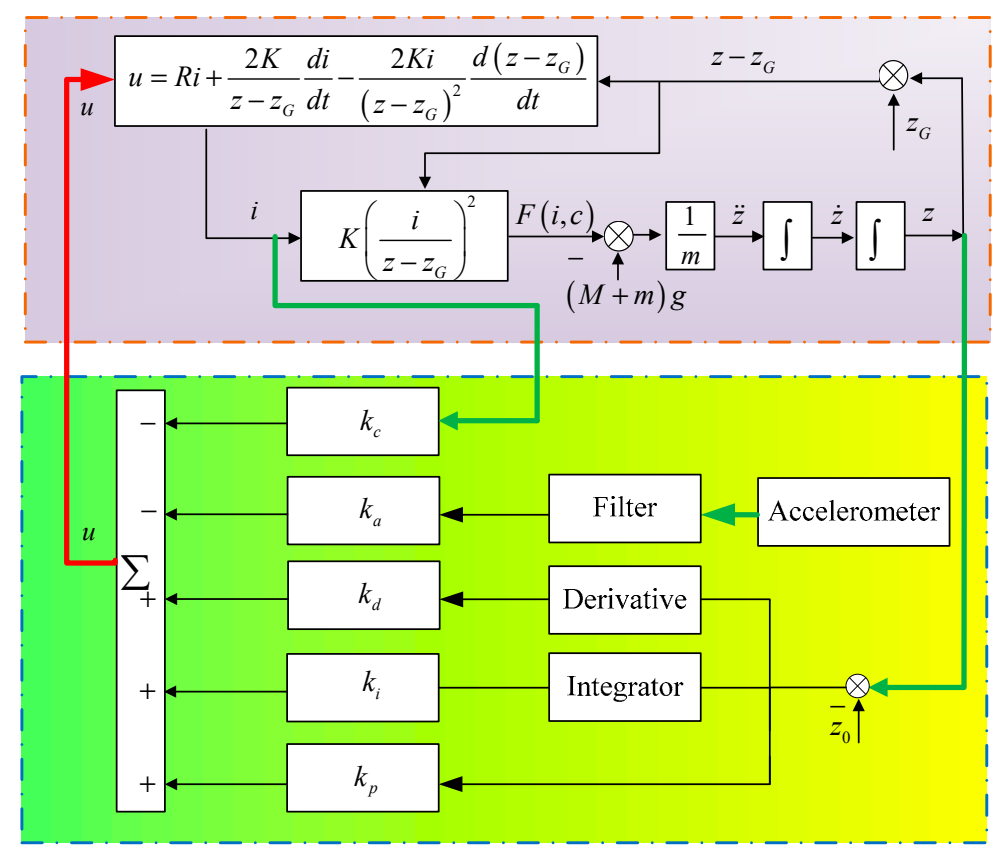

Figure 12. The new control block diagram of magnetic levitation system with consideration of vertical curve in maglev train line. 
When the maglev train passes through the track shown in Figure 8 at a certain speed, the magnetic levitation system by utilizing the new strategy will also go through four different stages: entering the concave curve stage, withdrawing from the concave curve stage, entering the convex curve stage and withdrawing from the convex curve stage. In order to achieve and investigate the tracking performance of the magnetic levitation system at different speeds, three representative speeds were also selected to observe the tracking performance on the above four stages (Figure 13).
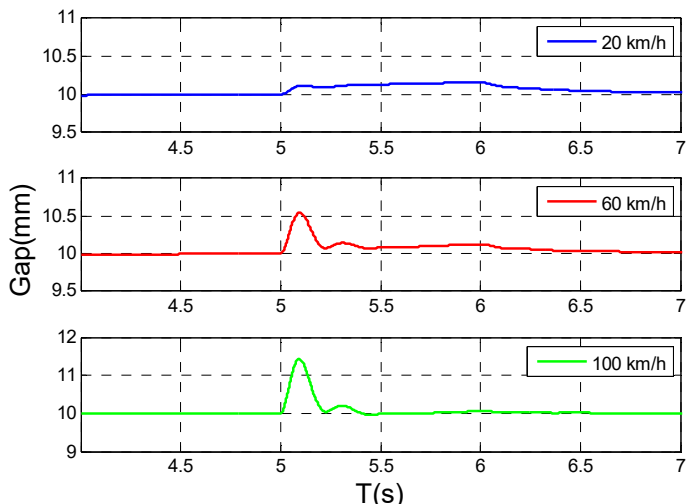

(a)
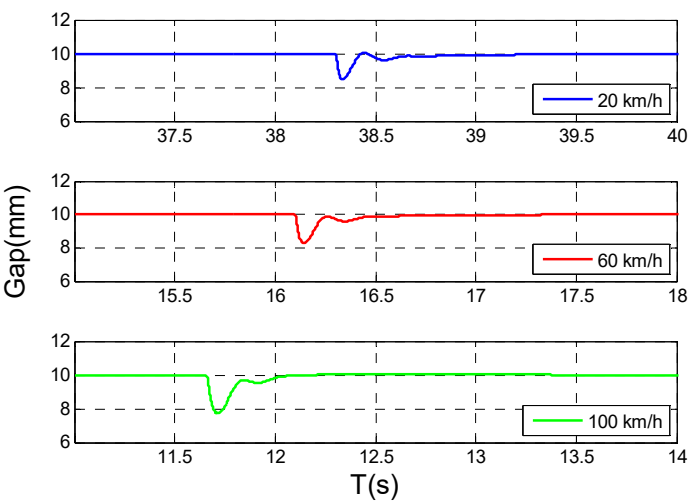

(c)
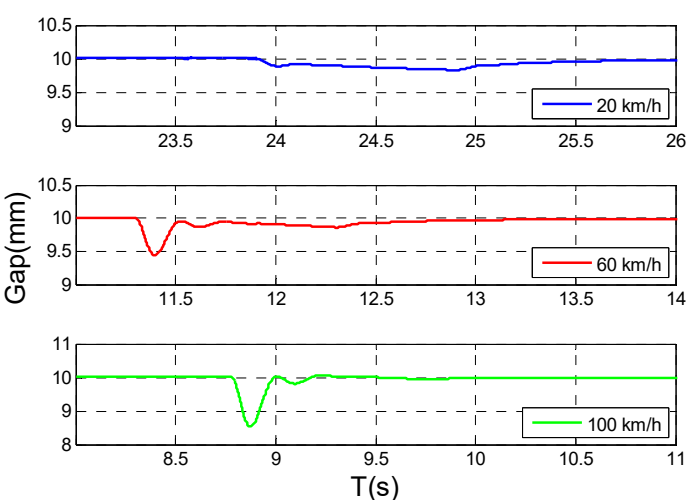

(b)
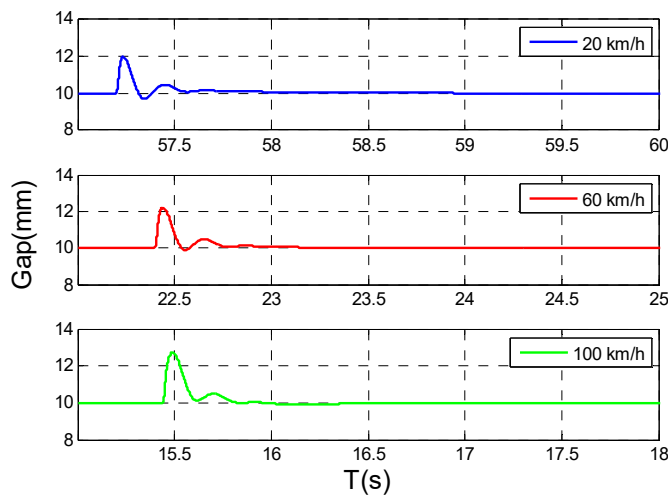

(d)

Figure 13. The tracking performance of magnetic levitation system by utilizing the new strategy when passing through the vertical track irregularity in maglev train line. (a) The suspension gap when entering the concave curve stage; (b) the suspension gap when withdrawing from the concave curve stage; (c) the suspension gap when entering the convex curve stage; (d) the suspension gap when withdrawing from the convex curve stage.

To illustrate the foregoing analysis and discussion, a number of significant simulations and investigations were performed to check the tracking performance of the magnetic levitation system when the vertical curve is passed. In order to test the validity, we performed several sets of comparative analyses. Compared with a nominal controller, the maximum fluctuations of the suspension gap at different speeds by utilizing the new strategy are described in Figure 14.

Our investigation has reached plenty of conclusions. The fluctuation of the suspension gap is obviously suppressed by utilizing the new strategy. The maximum reduction is nearly $1 \mathrm{~mm}$. Moreover, the maximum fluctuations of the suspension gap are all limited to less than $3 \mathrm{~mm}$. Therefore, the tracking performance of the magnetic levitation system by utilizing the new strategy improved the vertical track irregularity condition. 


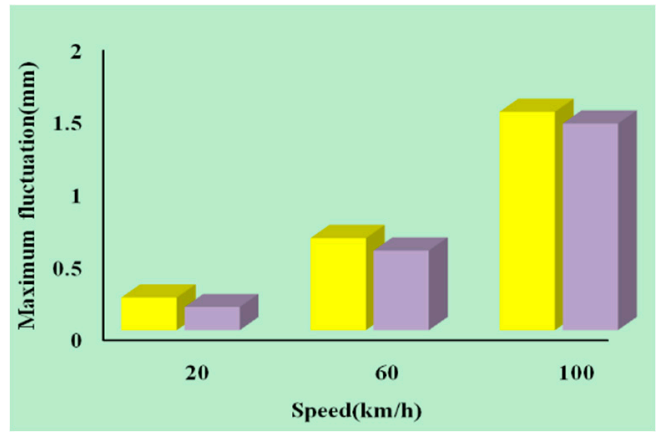

(a)

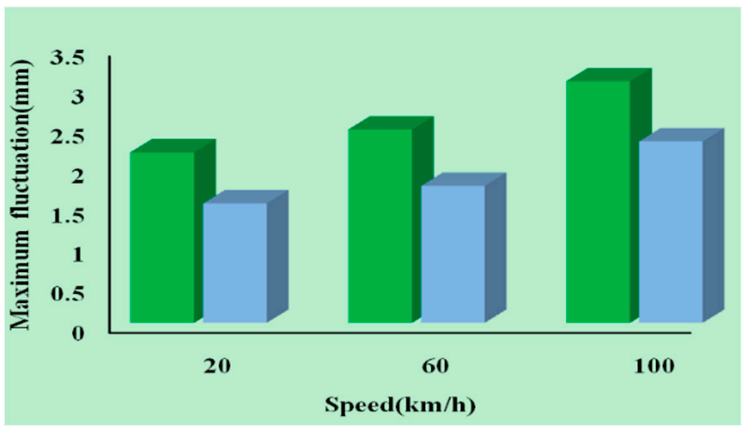

(c)

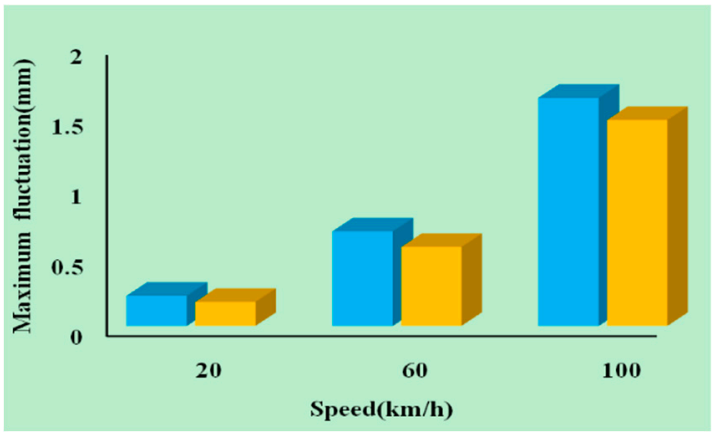

(b)

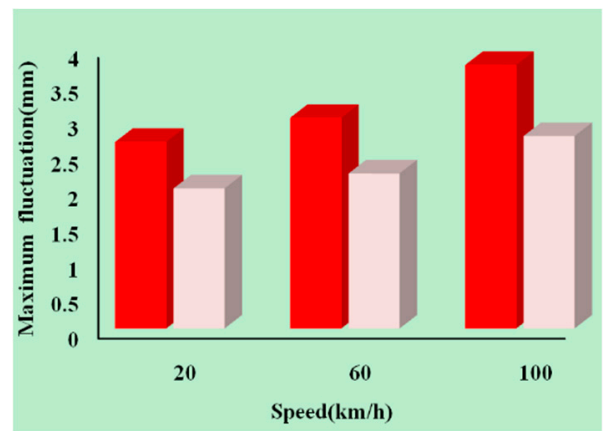

(d)

Figure 14. The contrastive maximum fluctuation of suspension gap when passing through the vertical track irregularity in maglev train line. (a) Entering the concave curve stage; (b) withdrawing from the concave curve stage; (c) entering the convex curve stage; (d) withdrawing from the convex curve stage.

\section{Implementation and Experiment}

We employed a model 4610-010 MEMS accelerometer with exceptional performance over a full operating temperature range of $-55^{\circ} \mathrm{C}$ to $+125^{\circ} \mathrm{C}$. As shown in Figure 15, this accelerometer has small size and low weight. The static and dynamic measurements are all feasible. In addition, the frequency response is taken from DC to $2700 \mathrm{~Hz}$.

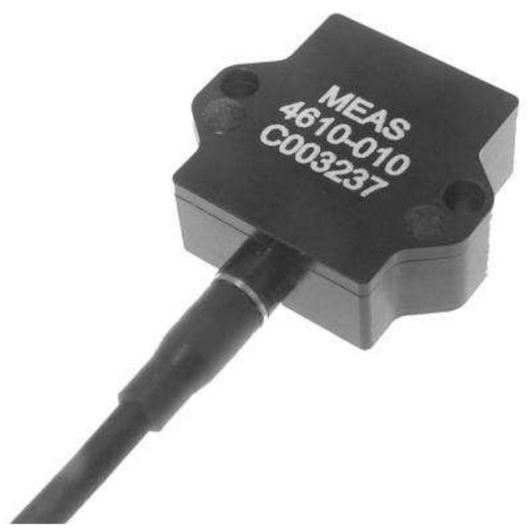

Figure 15. The photograph of model 4610-010 accelerometer.

The parameters of the model 4610-010 accelerometer are indicated in Table 2. 
Table 2. Parameter list of model 4610-010 accelerometer.

\begin{tabular}{ccc}
\hline Meaning & Quantity & Unit \\
\hline Range & \pm 10 & $\mathrm{~g}$ \\
Sensitivity & 200 & $\mathrm{mV} / \mathrm{g}$ \\
Frequency Response & $0-2000$ & $\mathrm{~Hz}$ \\
Residual Noise & 400 & $\mu \mathrm{RMS}$ \\
Non-Linearity (\%FSO) & \pm 0.1 & $/$ \\
Transverse Sensitivity & $<3$ & $\%$ \\
Damping Ratio & 0.7 & $/$ \\
Shock Limit & 6000 & $\mathrm{~g}$ \\
\hline
\end{tabular}

What's more, this strategy has already been successfully implemented and applied to the suspension controller for the magnetic levitation system in the Changsha maglev express. Figure 16 is a photograph of the suspension controller which was designed and manufactured by our team.

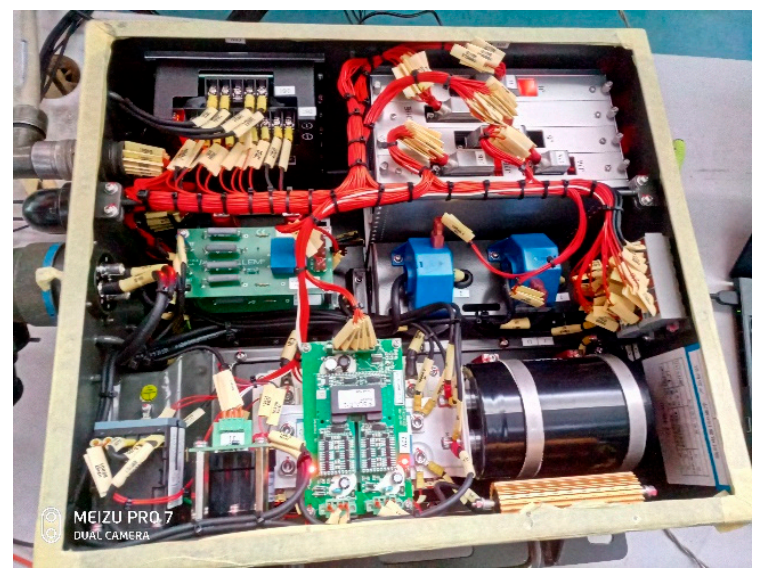

Figure 16. The photograph of designed and manufactured controller.

Since the Shanghai-Kunming high-speed railway passes through the middle of the maglev line, the maglev line has to cross under the high-speed railway line. As presented in Figure 17, the vertical track irregularity is similar to in Figure 8. When the maglev train passes through this section, the magnetic levitation system will also go through the above four different stages. The radius of the circular curve is $2000 \mathrm{~m}$ and the angle of slope is $4.1 \%$.

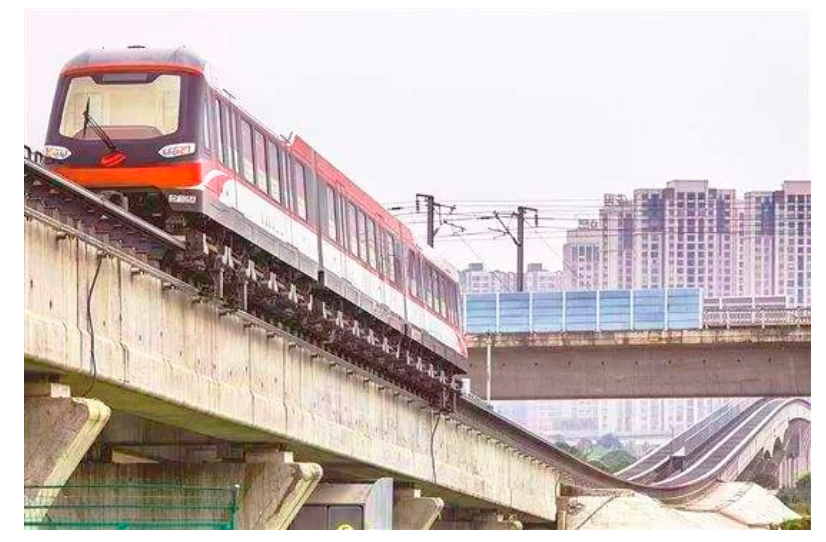

Figure 17. A photograph of the maglev train passing through the vertical section in Changsha maglev express. 
In order to observe the tracking performance of the maglev train passing through the above sections, the suspension gap and running speed need to be measured and recorded. The magnetic levitation system sends data to the on-board computer every $100 \mathrm{~ms}$ through the CAN network, and these data are stored in the on-board computer. In the Changsha maglev express line, the $\mathrm{Mc1}, \mathrm{M}$ and Mc2 maglev trains are organized into groups. Each maglev train has 20 sets of magnetic levitation systems. The suspension gap and speed of the tenth suspension point in each maglev train were selected and observed in Figure 18.
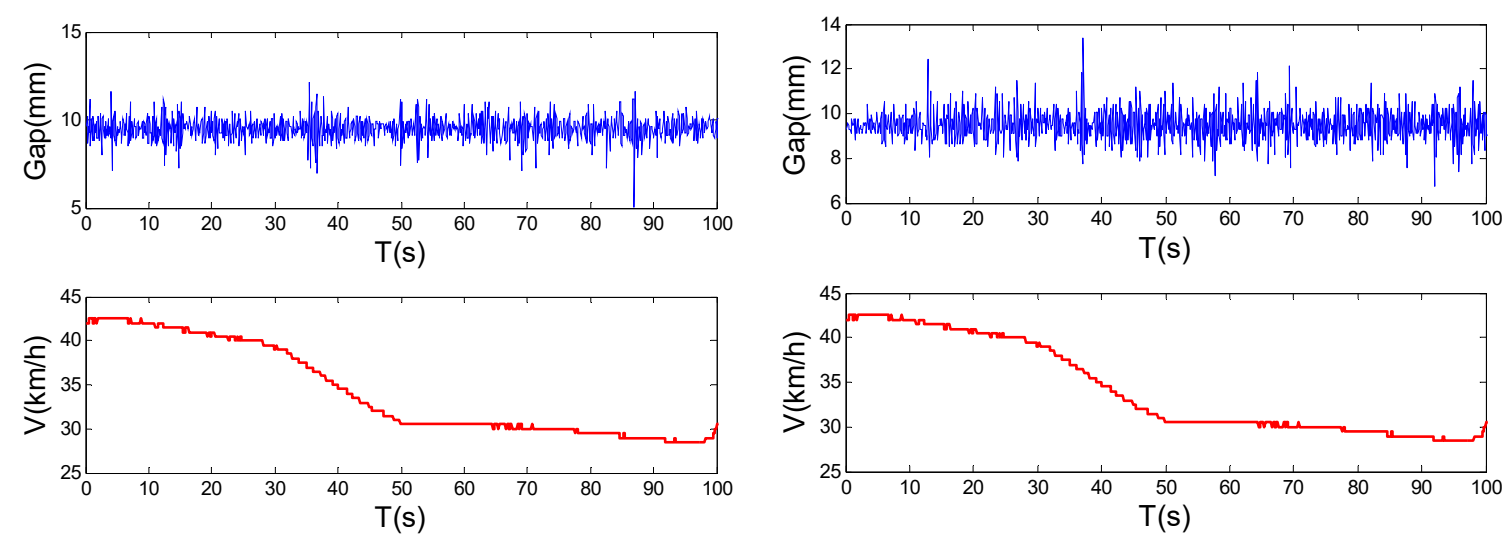

(a)

(b)
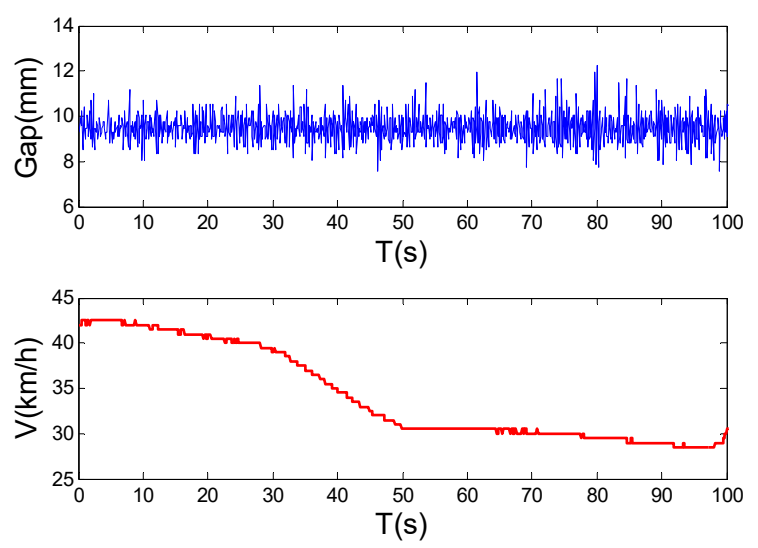

(c)

Figure 18. The suspension gap and speed of suspension point in the maglev train when the vertical section is passed. (a) The tenth suspension point in the Mc1 maglev train; (b) The tenth suspension point in the M maglev train; (c) The tenth suspension point in the Mc2 maglev train.

Based on analysis of the operation data, it was found that the performance of the actual suspension gap curve is different from that of the simulation curve. This is because the on-board computer only collects data from the magnetic levitation system every $100 \mathrm{~ms}$ in actual operation. The sampling frequency is too low to fully reflect the real-time dynamic characteristics of the suspension gap. What's more, the maglev train does not pass through the above section at a fixed speed in actual line operation, and the speed is constantly changing. However, it is obviously presented that the suspension gap always fluctuates up and down near the desired gap, and the maximum fluctuations of the suspension gap are almost all limited to less than $3 \mathrm{~mm}$, except the impression of noise. The fluctuations of the suspension gap are all in the reasonable range and the electromagnet doesn't collide with the track. In other words, the tracking performance of the magnetic levitation system by utilizing the new strategy was improved and qualified the vertical track condition. 


\section{Discussion}

In this paper, it is emphasized that the tracking performance of the magnetic levitation system is closely related to the running speed in the vertical track condition. An important and significant conclusion was acquired, that the fluctuation of suspension gap becomes more and more serious with the increase of speed. However, when the maglev train passes through the vertical curve, the tracking performance of the magnetic levitation system is also dictated by the radius of circular curve $r$ and the angle of slope $\alpha$.Therefore, the tracking performance of the magnetic levitation system in the different radiuses of circular curve and angles of slope need to be further analysis, calculation and verification. This will become a study emphasis in our further research.

\section{Conclusions}

In this paper, we established a mathematical model with consideration of vertical track irregularity to discuss and analyze the impact on the tracking performance of the magnetic levitation system. The tracking performance of the magnetic levitation system on the vertical track irregularity condition was investigated and achieved at different speeds. The investigation carried out by us has revealed that the fluctuation of suspension gap becomes more and more serious with the increase of running speed. For the benefit of improving the tracking performance of the magnetic levitation system, we proposed a new strategy by installing an accelerometer on the electromagnet as a solution to address this problem. What's more, this strategy has already been successfully implemented and applied to the suspension controller for a magnetic levitation system in the Changsha maglev express. The actual operation data demonstrates that the tracking performance of the magnetic levitation system was evidently improved. This fruitful work has produced an enormous application value.

Author Contributions: M.Z. is responsible for paper writing, modeling, algorithm design, simulation and experimental verification. X.L. is responsible for development and manufacture of controller. Z.L. is responsible for overall planning.

Funding: This research was funded by National Key R \& D Program of China, grant number 2016YFB1200601.

Acknowledgments: This work was supported by “National Key R \& D Program of China” under Grant 2016 YFB1200601.

Conflicts of Interest: The authors declare no conflict of interest.

\section{References}

1. Lee, H.W.; Kim, K.C.; Lee, J. Review of Maglev Train Technologies. IEEE Trans. Magn. 2006, 42, 1917-1925.

2. Lin, G.; Sheng, X. Application and further development of Maglev transportation in China. Transp. Syst. Technol. 2018, 4, 36-43. [CrossRef]

3. Gutierrez, H.M.; Luijten, H. 5-DOF Real-Time Control of Active Electrodynamic MAGLEV. IEEE Trans. Ind. Electron. 2018, 42, 1917-1925. [CrossRef]

4. Min, D.J.; Kwon, S.D.; Kwark, J.W.; Kim, M.Y. Gust Wind Effects on Stability and Ride Quality of Actively Controlled Maglev Guideway Systems. Shock Vib. 2017, 35, 1-23. [CrossRef]

5. Castellanos, M.; Miguel, L.; Galluzzi, R.; Bonfitto, A.; Tonoli, A.; Amati, N. Magnetic Levitation Control Based on Flux Density and Current Measurement. Appl. Sci 2018, 8, 2545. [CrossRef]

6. Shi, J.; Fang, W.S.; Wang, Y.J.; Zhao, Y. Measurements and analysis of track irregularities on high speed maglev lines. J. Zhejiang Univ. Sci. A 2014, 15, 385-394. [CrossRef]

7. Li, M.; Persson, I.; Spännar, J.; Berg, M. On the use of second-order derivatives of track irregularity for assessing vertical track geometry quality. Veh. Syst. Dyn. 2012, 50, 389-401. [CrossRef]

8. Zhao, X.; Li, Z.; Dollevoet, R. The vertical and the longitudinal dynamic responses of the vehicle-track system to squat-type short wavelength irregularity. Veh. Syst. Dyn. 2013, 51, 1918-1937. [CrossRef]

9. Yao, J.; Jiao, Z.; Ma, D.; Yan, L. High-Accuracy Tracking Control of Hydraulic Rotary Actuators with Modeling Uncertainties. IEEE/ASME Trans. Mechatron. 2014, 19, 633-641. [CrossRef]

10. Ding, Z.; Li, W.; An, J.; Hao, 1.; Zhang, Q. Adaptive fuzzy fault-tolerant control with guaranteed tracking performance for nonlinear strict-feedback systems. Fuzzy Sets Syst. 2016, 302, 82-100. 
11. Kim, W.; Shin, D.; Chung, C. The Lyapunov-based controller with a passive nonlinear observer to improve position tracking performance of microstepping in permanent magnet stepper motors. Automatica 2012, 48, 3064-3074. [CrossRef]

12. Han, S.; Lee, J. Recurrent fuzzy neural network backstepping control for the prescribed output tracking performance of nonlinear dynamic systems. ISA Trans. 2014, 53, 33-43. [CrossRef] [PubMed]

13. Bagheri, A.; Karimi, T.; Amanifard, N. Tracking performance control of a cable communicated underwater vehicle using adaptive neural network controllers. Appl. Soft Comput. 2010, 10, 908-918. [CrossRef]

14. Tan, W. Unified Tuning of PID Load Frequency Controller for Power Systems via IMC. IEEE Trans. Power Syst. 2010, 25, 341-350. [CrossRef]

15. Lee, J.S.; Choi, S.; Kim, S.S.; Park, C.; Kim, Y.G. A Mixed Filtering Approach for Track Condition Monitoring Using Accelerometers on the Axle Box and Bogie. IEEE Trans. Instrum. Meas. 2012, 61, 749-758. [CrossRef]

(C) 2019 by the authors. Licensee MDPI, Basel, Switzerland. This article is an open access article distributed under the terms and conditions of the Creative Commons Attribution (CC BY) license (http://creativecommons.org/licenses/by/4.0/). 